\title{
Statins and metachronous recurrence after endoscopic resection of early gastric cancer: a nationwide Korean cohort study
}

\author{
Hyunsoo Chung ${ }^{1,2} \cdot$ Hyun Ju Kim ${ }^{1,2,3} \cdot$ Hyun Chae Jung ${ }^{1} \cdot$ Sang Kil Lee ${ }^{4}$ Sang Gyun Kim ${ }^{1} \mathbb{C}$
}

Received: 20 June 2019 / Accepted: 10 January 2020 / Published online: 24 January 2020

(c) The International Gastric Cancer Association and The Japanese Gastric Cancer Association 2020

\begin{abstract}
Background Statins have shown to reduce the risk of various cancers. However, their effects on metachronous recurrence (MR) after endoscopic resection (ER) for early gastric cancer (EGC) are unknown. We evaluate their effects on MR development after ER for EGC.

Methods We selected 11,568 patients who received ER for EGC from 2002 to 2011 from the Korean National Health Insurance database and classified into 2 groups: control and statins using propensity score matching. Metachronous recurrence was defined as the second ER or gastrectomy performed 6 months after the first ER.

Results Mean follow-up period was $8.8 \pm 3.1$ years. Statins showed a significantly lower incidence of MR than the control group (12.5\% vs $2.2 \%$, respectively, $P<0.01)$. After conducting competing risk analyses and time-dependent cox regression analysis considering immortal time bias, statins still showed a lower incidence rate of MR compared to that observed in the control group. For the multivariate analysis, statins remained significant (HR 0.17 ; 95\% CI $0.13-0.24, P<0.01$ ). In the dose-response analysis, an inverse dose-response relationship was identified between MR and statins $(P<0.01)$.

Conclusion Statins was significantly associated with a reduced risk of MR after ER for EGC with an inverse dose-response relationship.
\end{abstract}

Keywords Medication - Recurrence $\cdot$ Stomach neoplasm - Endoscopic treatment

\begin{tabular}{|c|c|c|c|c|}
\hline \multicolumn{3}{|c|}{ Abbreviations } & DDD & Defined daily dose \\
\hline \multirow{3}{*}{\multicolumn{2}{|c|}{$\begin{array}{l}\text { ADDD } \\
\text { BMI } \\
\text { CI }\end{array}$}} & Average DDD & EGC & Early gastric cancer \\
\hline & & Body mass index & EMR & Endoscopic mucosal resection \\
\hline & ER & Endoscopic resection \\
\hline & & & ESD & Endoscopic submucosal dissection \\
\hline \multicolumn{3}{|c|}{$\begin{array}{l}\text { Hyunsoo Chung and Hyun Ju Kim have contributed equally to this } \\
\text { work. }\end{array}$} & $\begin{array}{l}\text { H. pylori } \\
\text { HR }\end{array}$ & $\begin{array}{l}\text { Helicobacter pylori } \\
\text { Hazard ratio }\end{array}$ \\
\hline \multirow[t]{2}{*}{ 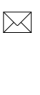 } & \multirow{2}{*}{\multicolumn{2}{|c|}{$\begin{array}{l}\text { Sang Kil Lee } \\
\text { sklee@yuhs.ac }\end{array}$}} & & revision \\
\hline & & & KCCR & Korean Central Cancer Registry \\
\hline \multirow[t]{2}{*}{$\triangle$} & \multirow{2}{*}{\multicolumn{2}{|c|}{$\begin{array}{l}\text { Sang Gyun Kim } \\
\text { harley1333@hanmail.net }\end{array}$}} & NHIS & National Health Insurance Service \\
\hline & & & MR & Metachronous recurrence \\
\hline \multirow[t]{3}{*}{1} & \multicolumn{2}{|c|}{ Department of Internal Medicine and Liver Research } & OR & Odds ratio \\
\hline & \multirow{2}{*}{\multicolumn{2}{|c|}{$\begin{array}{l}\text { Institute, Seoul National University College } \\
\text { of Medicine, Dahangno 101, Jongno-gu, Seoul 110-744, }\end{array}$}} & PSM & Propensity score-matching \\
\hline & & & $\mathrm{SD}$ & Standard deviation \\
\hline
\end{tabular}

\section{Introduction}

Endoscopic resection (ER), including endoscopic submucosal dissection (ESD) and endoscopic mucosal resection (EMR), are widely used treatments for gastrointestinal 
neoplasia. ER is safe, less invasive, and effective for early gastric cancer (EGC); however, the risk of metachronous recurrence (MR) is higher in patients who undergo ER than in those who undergo surgery [1-4]. The 5-year MR rate after ER for EGC varies from 3.6 to $16 \%$ [2, 3, 5-8], and known risk factors are old age, cigarette smoking, male sex, multiple initial EGCs, mucosal atrophy, intestinal metaplasia, and failure of Helicobacter pylori (H. pylori) eradication $[5,6,9-11]$.

Recently, Statins have attracted widespread attention for their potential anti-cancer and/or anti-inflammatory effects in cancers, such as renal, colon, lung, skin, prostate, non-Hodgkin's lymphoma, and hepatocellular carcinoma [12-14]. Statins, in particular, have shown to reduce gastric cancer risk [15-18].

However, to the best of our knowledge, no studies have investigated statins' protective effects on MR after ER for EGC.

The aim of this retrospective, population-based cohort study is to clarify the potential protective effects of statins on the incidence of MR after ER for EGC using information from the Korean National Health Insurance Service (NHIS) database.

\section{Materials and methods}

\section{Data sources}

The NHIS is a mandatory universal health insurance system and the only one available in Korea; it has provided comprehensive medical care to more than $98 \%$ of all Korean citizens since 1999 [19, 20]. The NHIS database contains information on Qualification, Claim, Health check-up, and death; therefore, the NHIS database can be used to conduct population-based, nationwide studies for various diseases [21]. Moreover, the Korean Central Cancer Registry (KCCR), which is a part of the NHIS database and used in this study, is very accurate and the completeness of cancer incidence data was estimated to be $97.8 \%$ [22]. This registry is regularly managed and verified by multiple national government agencies such as the Ministry of Health and Welfare, Ministry of Strategy and Finance, and Statistics Korea, because the registered patients receive benefits to only pay $5 \%$ of the total medical expenses for 5 years after cancer registration [23].

The detailed information on the NHIS database and list of publications using this database could be obtained from the NHIS website [21].

We evaluated general health check-up, lifetime transition period health check-up, and cancer check-up from the Health check-up database. All examinees were requested to have biannual health check-ups. The proportion of complete health check-ups was $68 \%$ in 2013. By evaluating all aspects in the Health Checkup database and combining them with the claims database, the lack of laboratory and personal history data can be overcome. Furthermore, long-term follow-up of a single individual will allow us to perform longitudinal studies of casual relationships [24]. This study was approved by the Institutional Review Board of the Seoul National University Hospital and Yonsei University College of Medicine (Institutional Review Board No. E-1704-046-844). Informed consent was waived, because the study was based on routinely collected administrative data, and patient data were kept anonymous.

\section{Study design}

This population-based observational cohort study aimed to investigate the effect of statins on MR of gastric intraepithelial neoplasm after ER of EGC. The NHIS database from January 2002 to October 2016 was reviewed for outcome analysis and the database from January 2002 to December 2011 was used to identify the study cohort to secure a minimum follow-up duration of 5 years. ICD-10 was used to identify gastric cancer (C16.00-C16.99), gastric benign neoplasm (D13.1), diabetes (E10-14), and hyperlipidemia (E78.0-78.5) [25, 26]. We used procedural codes for ESD (ZQ933, ZX704), EMR (Q7652, QX701), and gastrectomy (Q0251-Q0259, Q2594, Q2598, QA536, Q2533-2537). Patients who previously underwent gastrectomy or ER and those who underwent ER for gastric adenoma were excluded. Overall, 12,589 patients had claimed payments for a first ER for EGC from January 2002 to December 2011. Among them, 132 were excluded due to inaccurate information $(n=37)$ and claimed payments for both ER and gastrectomy from the same account $(n=95)$. In addition, 889 patients were excluded for synchronous recurrence or incomplete resection, because ER or gastrectomy was claimed within 6 months from the first ER for EGC. In total, 11,568 patients were included in the final cohort (Fig. 1).

\section{Statins exposure}

The Statin groups included patients who had claimed payments for statins, respectively, after the first ER, and the control group included patients who had never claimed payments for statins after first ER. Korean drug codes were used to classify statins (atorvastatin, rosuvastatin, simvastatin, cerivastatin, fluvastatin, lovastatin, pitavastatin, and pravastatin).

\section{Defined daily dose (DDD) and average defined daily dose (ADDD)}

To examine the dose-effect relationship between drug use and MR, we used DDD and ADDD. DDD is a unit for 
Fig. 1 Enrolment of patients. Overall, 12,589 patients had claimed payments for a first ER for EGC from January 2002 to December 2011. After exclusion, in total, 11,568 patients were included in the final cohort. $E G C$ early gastric cancer, NHIS National Health Insurance Service, ESD endoscopic submucosal dissection
Endoscopic treatment for EGC from January 2002 to December 2011 from NHIS claim database ( $n=12,589)$

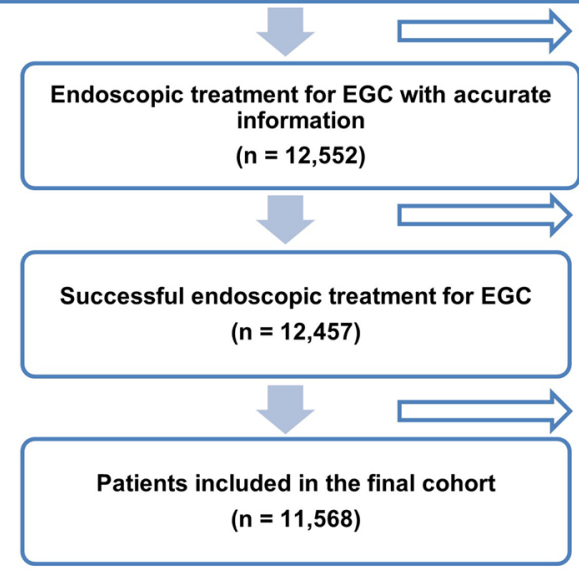

Inaccurate information $(n=37)$

Patients had claimed for ESD and operation in same account $(n=95)$

Recurrence within 6 months $(\mathrm{n}=889$ ) measuring a prescribed amount of drug. According to the World Health Organization, it is defined as "the assumed average maintenance dose per day of a drug consumed for its main indication in adults" [27]. For example, 1 DDD is equivalent to a single dose of $30 \mathrm{mg}$ simvastatin or $20 \mathrm{mg}$ atorvastatin. Cumulative DDD is the total number of drug pills times dose per tablet divided by DDD, and ADDD is cumulative DDD divided by follow-up time.

\section{Covariates}

The covariates that could plausibly confound associations between statin use and MR are as follows: age at diagnosis, sex, body mass index (BMI) at the time closest to and preceding diagnosis, smoking status, diabetes mellitus, and dyslipidemia, and dosage of statin. In the present study, the effect of $H$. pylori could not be analyzed, because the NHIS database only contains claims on drugs and laboratory prescriptions. Therefore, we were unable to study to results of $H$. pylori eradication.

\section{Outcome measures}

Outcome measurement was defined as the incidence of MR of gastric intraepithelial neoplasms. MR was defined as claims for ER or gastrectomy $>6$ months after the first ER for EGC. We excluded patients who underwent ER or gastrectomy for gastric polyps, gastric subepithelial tumors, and peptic ulcer perforation, and only included those patients who had the disease code of gastric cancer or gastric adenoma registered at the time of the second claim.

\section{Statistical analysis}

Descriptive analyses were performed to delineate the characteristics of control and statin groups using the $t$ test and Chi-square test. To compare the risk of MR with a matched population, we conducted propensity score matching (PSM) (control vs statin). A propensity score was calculated for each patient using a logistic regression model. The PSM model consisted of age, sex, BMI, diabetes, and follow-up duration; the nearest neighbor matching with the propensity score of each patient was used for $1: 1$ matching.

To adjust for death, which is a potential competing risk, we performed competing risk analysis using a semiparametric proportional hazard model for cumulative incidence of recurrence [28].

To avoid immortal time bias, we performed timedependent cox regression analysis, which considered person-time before first statin prescription as unexposed [29]. Furthermore, sensitivity analysis conducted which assess statin exposure for the first 6 months as non-exposure to deal with the possibility that the effect of statin was overestimated.

In addition, Cox proportional hazard models with Efron's method were used to calculate the hazard ratio (HR) of MR. Adjusted HR and 95\% confidence intervals (CI) in the multivariable Cox regression were calculated with adjustments for age, sex, BMI, and smoking. All statistical analyses were performed using the SAS version 9.2 software (SAS Institute, Inc., Cary, NC, USA) and the R Project for Statistical Computing (version 3.3.1; R Foundation for Statistical Computing, Vienna, Austria). A $P$ value of $<0.05$ was considered to be statistically significant. 


\section{Results}

\section{Clinical characteristics after PSM analysis}

All included patients $(n=11,568)$ were divided into 2 groups based on drug use as control $(n=2319)$ and stain $(n=2319)$, after conducting propensity matching. Patients' baseline characteristics are presented in Table 1. The mean age was 63.0 years [standard deviation (SD), 9.9 years] and mean BMI was $24.1 \mathrm{~kg} / \mathrm{m}^{2}\left(\mathrm{SD}, 2.9 \mathrm{~kg} / \mathrm{m}^{2}\right)$. The mean followup duration was 8.8 years (SD, 3.1 years). Overall, patients were more likely to be non-smoking men. The MR rate was $7.3 \%$, and it was lower in statin group than in the control group ( $12.5 \%$ vs $2.2 \%$, respectively, $P<0.01$ ).

\section{Statin exposure and MR after ER of EGC}

The log-rank test also confirmed the difference in cumulative MR between the two groups $(12.5 \%$ vs. $2.2 \%$,
$P<0.01)$; after competing risk analyses according to the methods described above, we observed that statin group still showed a lower incidence rate of MR compared to that observed in the control group $(P<0.01)$. In the timedependent cox regression analysis considering immortal time bias and additional analysis except for the initial 6 months statin exposure effect, statin group still showed a lower incidence rate of MR compared to that observed in the control group $(P<0.01)$ (Fig. 2).

\section{Factors associated with MR}

In PSM (control vs statin), age and BMI were associated with a high MR rate (HR 1.02; 95\% CI 1.01-1.03; $P<0.01$, HR $1.04 ; 95 \%$ CI $1.00-1.08 ; P=0.03$ ), and female sex and statin use were associated with a low MR rate (HR 0.73; 95\% CI 0.57-0.95; $P=0.021$, HR 0.17; 95\% CI 0.13-0.24; $P<0.01$ ) (Table 2).
Table 1 Baseline characteristics of patients after propensity score matching (1:1 matching)

\begin{tabular}{|c|c|c|c|c|c|}
\hline & $n$ & $\begin{array}{l}\text { Total } \\
(n=4638)\end{array}$ & $\begin{array}{l}\text { Control } \\
(n=2319)\end{array}$ & $\begin{array}{l}\text { Statin } \\
(n=2319)\end{array}$ & $P$ \\
\hline Age, years & 4638 & $63.0 \pm 9.9$ & $62.9 \pm 10.4$ & $63.0 \pm 9.4$ & 0.784 \\
\hline Sex & 4638 & & & & 0.172 \\
\hline Female & & $1569(33.8 \%)$ & $762(32.9 \%)$ & $807(34.8 \%)$ & \\
\hline Male & & $3069(66.2 \%)$ & $1557(67.1 \%)$ & $1512(65.2 \%)$ & \\
\hline BMI, $\mathrm{Kg} / \mathrm{m}^{2}$ & 4638 & $24.1 \pm 2.9$ & $24.1 \pm 2.9$ & $24.2 \pm 2.9$ & 0.563 \\
\hline Smoking & 4563 & & & & 0.073 \\
\hline Current & & $868(19.0 \%)$ & $404(17.7 \%)$ & $464(20.3 \%)$ & \\
\hline Former & & $806(17.7 \%)$ & $413(18.1 \%)$ & $393(17.2 \%)$ & \\
\hline Never & & $2889(63.3 \%)$ & $1465(64.2 \%)$ & $1424(62.4 \%)$ & \\
\hline $\mathrm{DM}$ & 4638 & & & & 0.698 \\
\hline No & & $4529(97.6 \%)$ & $2262(97.5 \%)$ & $2267(97.8 \%)$ & \\
\hline Yes & & $109(2.4 \%)$ & $57(2.5 \%)$ & $52(2.2 \%)$ & \\
\hline Hyperlipidemia & 4638 & & & & $<0.01$ \\
\hline No & & $2244(96.9 \%)$ & $2244(96.8 \%)$ & $0(0.0 \%)$ & \\
\hline Yes & & $2394(51.6 \%)$ & $75(3.2 \%)$ & $2319(100 \%)$ & \\
\hline Creatinine, mg/daysL & 1587 & $1.0 \pm 0.9$ & $1.0 \pm 0.7$ & $1.1 \pm 1.1$ & 0.017 \\
\hline Follow-up, years & 4638 & $8.8 \pm 3.1$ & $8.8 \pm 3.2$ & $8.8 \pm 3.0$ & 0.773 \\
\hline recur & 4638 & & & & $<0.01$ \\
\hline No & & $4298(92.7 \%)$ & $2030(87.5 \%)$ & $2268(97.8 \%)$ & \\
\hline Yes & & $340(7.3 \%)$ & $289(12.5 \%)$ & $51(2.2 \%)$ & \\
\hline Recurrence type & 340 & & & & 0.066 \\
\hline Adenoma & & $106(31.2 \%)$ & $84(29.1 \%)$ & $22(43.1 \%)$ & \\
\hline Cancer & & $234(68.8 \%)$ & $205(70.9 \%)$ & $29(56.9 \%)$ & \\
\hline Alive & 4638 & & & & $<0.01$ \\
\hline No & & $549(11.8 \%)$ & $344(14.8 \%)$ & $205(8.8 \%)$ & \\
\hline Yes & & $4089(88.2 \%)$ & $1975(85.2 \%)$ & $2114(91.2 \%)$ & \\
\hline
\end{tabular}

$B M I$ body mass index, $D M$ diabetes mellitus 

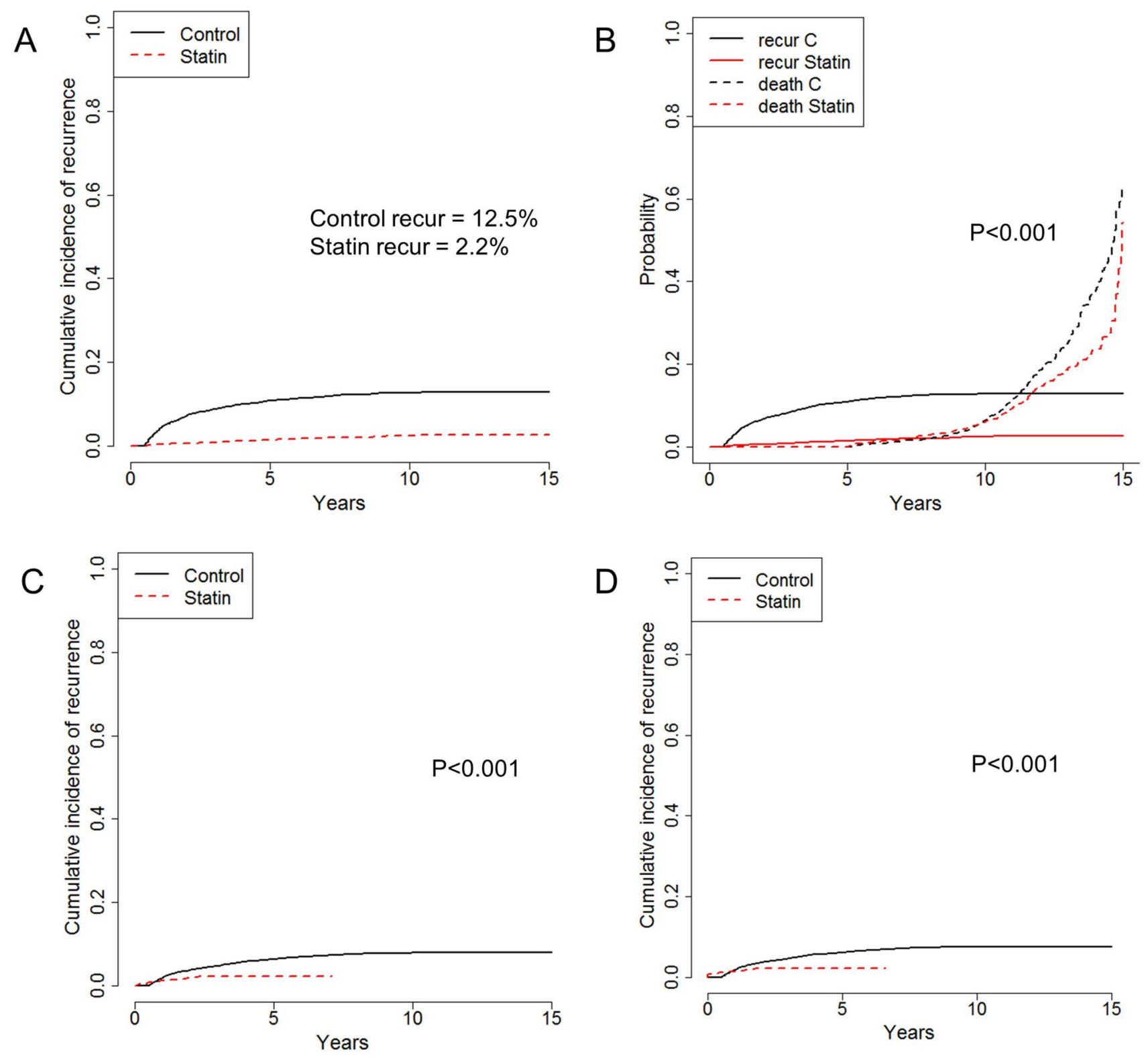

Fig. 2 Cumulative MR according to study group. The log-rank test (a), competing risk analysis (b), time-dependent cox regression analysis considering immortal time bias (c), and sensitivity analysis

except for the initial 6 months statin exposure effect (d). In the all analysis, statin group showed a lower incidence rate of MR compared to that observed in the control group $(P<0.01)$

\section{Dose-response relationship}

\section{Discussion}

In the dose-response analysis using ADDD, a significant inverse dose-response relationship was observed between MR rate and statin use $(P<0.01)$ (Table 3$)$.

This population-based, nationwide, retrospective cohort study using the NHIS database demonstrated that statin was associated with a reduced incidence of MR in patients 
Table 2 Relative risk of MR after endoscopic treatment for EGC

\begin{tabular}{|c|c|c|c|c|c|c|}
\hline & \multicolumn{6}{|c|}{ Control vs statin } \\
\hline & \multicolumn{3}{|c|}{ Unadjusted } & \multicolumn{3}{|c|}{ Adjusted } \\
\hline & HR & $95 \%$ CI & $P$ & HR & $95 \%$ CI & $P$ \\
\hline \multicolumn{7}{|l|}{ Sex } \\
\hline Male & 1.00 & & & 1.00 & & \\
\hline Female & 0.81 & $0.64-1.02$ & 0.072 & 0.73 & $0.57-0.95$ & 0.021 \\
\hline Age & 1.02 & $1.01-1.03$ & 0.01 & 1.02 & $1.01-1.03$ & $<0.01$ \\
\hline BMI & 1.03 & $0.99-1.07$ & 0.099 & 1.04 & $1.00-1.08$ & 0.03 \\
\hline \multicolumn{7}{|l|}{ Smoking } \\
\hline Current & 1.00 & & & 1.00 & & \\
\hline Former & 0.92 & $0.63-1.32$ & 0.639 & 0.84 & $0.58-1.21$ & 0.351 \\
\hline Never & 1.02 & $0.77-1.34$ & 0.913 & 1.05 & $0.77-1.42$ & 0.753 \\
\hline Statin use & 0.17 & $0.13-0.23$ & $<0.01$ & 0.17 & $0.13-0.24$ & $<0.01$ \\
\hline
\end{tabular}

BMI body mass index

${ }^{\mathrm{a} C}$ Covariates are sex, age, BMI, and smoking
Table 3 Average defined daily dose of statin and metformin and the risk for recurrence

\begin{tabular}{lll}
\hline & \multicolumn{2}{l}{ Matched cohort $(n=4644)$} \\
\cline { 2 - 3 } & $\begin{array}{l}\text { Statin } \\
\text { HR }(95 \% \mathrm{CI})\end{array}$ & $P$ \\
\hline Statin & & \\
Control & 1.00 & $<0.01$ \\
$0<\mathrm{ADDD} \leq 0.05$ & $0.27(0.20-0.38)$ & $<0.01$ \\
$0.05<\mathrm{ADDD} \leq 0.1$ & $0.05(0.03-0.11)$ & \\
\hline
\end{tabular}

$A D D D$ average $\mathrm{DDD}=$ cumulative $\mathrm{DDD} /$ follow-up time, cumulative $D D D$ total number of drug pills $\times$ dose per tablet/defined daily dose, $D D D$ defined daily dose, $H R$ hazard ratio

All HRs were adjusted for age, sex, BMI, and smoking

who underwent ER for EGC. Furthermore, significant inverse dose-response relationships were observed in statin-using groups.

Previously, several studies have demonstrated the protective effects of statins on gastric cancer.

Chiu et al. performed a population-based case-control study, which revealed that ever-use of any statins was associated with a significant decrease in the risk of gastric cancer $(\mathrm{OR}=0.68,95 \% \mathrm{CI}=0.49-0.95)$ [16]. In addition, meta-analysis of studies revealed that statins use and gastric cancer risk were dose-dependent in Asian and western populations [17]. Lee et al. [15] used an exactmatching case-control design and showed that statins use is inversely associated with gastric cancer risk $(\mathrm{OR}=0.18$; 95\% CI $=0.14-0.24 ; P<0.0001)$.

Experimental studies have demonstrated that the protective effects of statins in these studies are related to the principle of promoting apoptosis and inhibiting proliferation of gastric cancer cells [30, 31].

As stated above, most previous studies have focused on the risk of gastric cancer in patients using statins. To the best of our knowledge, ours is the only observational study that demonstrates the efficacy of statins on MR after ER for EGC. This study is a large cohort study involving more patients than previously reported, with a median follow-up period of 8.8 years (SD 3.1 years), which allowed meaningful recurrence analysis. Using the Prospective Prescription Record of NHIS, the recall bias was reduced compared to that with self-reported medication use and the measurement error with drug exposure was minimized compared to that with single- or multi-center studies.

In addition, propensity score matching was performed to minimize the difference between the groups, and to adjust for death, which is a potential competing risk, a competing risk analysis using a semiparametric proportional hazards model for cumulative incidence of the recurrence was performed [28]. In addition, we tried to avoid immortal time bias and the possibility that the effect of statin was overestimated, which may exaggerate associations[29]. Furthermore, we used time-varying nature of drug exposures (ADDD) for the sensitivity analyses of dose-response associations between statin and MR.

This study has some limitations. The risk factors for MR after endoscopic treatment for EGC are known as age, male sex, cigarette smoking, multiple initial EGCs, mucosal atrophy, intestinal metaplasia, and failure of $H$. pylori eradication [5, 6, 9-11]. Our data also demonstrated that age, male sex, and high BMI increase MR after the first ER for EGC. However, the effect of risk factors, such as $H$. pylori infection, and endoscopic findings, such as multiple initial EGCs, mucosal atrophy, and intestinal metaplasia, which 
are not available in the NHIS claim database, could not be analyzed. As in the previous study [32], the results of $H$. pylori eradication were not indicated on the NHIS claims, which is a limitation. However, we found no statistically significant difference in the prescription rate of $H$. pylori eradication among groups in this study. Therefore, it can be assumed that the infection rate among the group is not significantly different.

In addition, coding errors are possible in this kind of huge nationwide database. However, there have been several sophisticated studies using the NHIS database with the same coding method as this study $[25,32]$ and the KCCR is highly accurate. In addition, endoscopic procedures and prescriptions for medicines are also paid after the screening of a health insurance review and an assessment service based on data requested by medical institutions, so that coding errors are expected to be low [8]. The diagnosis of MR was based on the insurance database that indicated ER or gastrectomy recurrence $>6$ months after the first ER for EGC. The diagnosis and stage of recurrent disease was not clear. In addition, there might be some patients who did not receive additional treatment for MR due to several reasons (e.g., far advanced staged and poor general conditions); therefore, it is possible that the MR rate might be slightly underestimated. However, the rate of extragastric recurrence after ESD for EGC is very low and ER is a minimally invasive procedure, which allows for conscious sedation. Therefore, the effect of the patient's condition on the results is considered minimal. In addition, patients within the statin groups could have poorer health conditions that require more frequent clinic visits and may be more likely to undergo tests or procedures. It is, therefore, possible that these patients were diagnosed with an early disease stage and lower risk of recurrence, compared to patients with no other comorbidities who may have presented at a later stage with a greater risk of recurrence.

Since our study is based on a nationwide cohort that utilized a code database, it is difficult to distinguish between local recurrence and MR which is another limitation. However, we considered that local recurrence of $1-2 \%$ did not significantly affect our findings $[1,3,4,8]$. Although we could not accurately determine the interval and frequency of follow-up endoscopy, in most hospitals, follow-up endoscopy and abdomen/pelvic computed tomography after ESD for EGC were performed 1-2 times a year for 5 years and an annual follow-up endoscopy was recommended after 5 years.

Although prior statins use before the first ER may have affected the results, the focus of our study was the statins effect had on MR after first ER; therefore, we only included the amount of statins after the first ER. To observe the effects of statins prior to the first ER, it is necessary to have an accurate starting point indicating when the medicine was initiated, but the NHIS claim database did not provide such information. Since the NHIS indicated the number of filled prescriptions, such data might not reflect the actual dose taken by the patients. We presumed that all medications were taken by the patients as prescribed, which could overestimate the actual ingested dosage. However, statins can only be obtained with a prescription in Korea, so the difference between the actual dose and the prescription dose will not be substantial, compared to over-the-counter drugs.

Finally, Koreans have a higher rate of gastric cancer than Westerners; thus, the applicability of our findings to the Western population is limited.

Despite these limitations, our results are remarkable, because we used statins in a large population using the NHIS claim database, to study the risk of metachronous gastric cancer after endoscopic treatment with EGC.

In conclusion, this study shows that statins may be independent chemopreventive agents, with dose-response effects in reducing MR of gastric intraepithelial neoplasm after ER of EGC. To confirm the antitumor effects of statins, it is necessary to investigate the efficacy, minimum effective dose, starting time, proper period of use, side effects, and response in cancer patients without hyperlipidemia by conducting a prospective randomized-controlled trial.

Acknowledgements The authors thank the staff of the National Health Insurance Service.

\section{Compliance with ethical standards}

Conflict of interest The authors declare that they have no association with or financial interest in any commercial company relevant to this study.

Ethical approval This article does not contain any studies with human participants or animals performed by any of the authors.

\section{References}

1. Park CH, Lee H, Kim DW, Chung H, Park JC, Shin SK, et al. Clinical safety of endoscopic submucosal dissection compared with surgery in elderly patients with early gastric cancer: a propensitymatched analysis. Gastrointest Endosc. 2014;80(4):599-609.

2. Ryu SJ, Kim BW, Kim BG, Kim JH, Kim JS, Kim JI, et al. Endoscopic submucosal dissection versus surgical resection for early gastric cancer: a retrospective multicenter study on immediate and long-term outcome over 5 years. Surg Endosc. 2016;30(12):5283-9.

3. Pyo JH, Lee H, Min BH, Lee JH, Choi MG, Lee JH, et al. Longterm outcome of endoscopic resection vs surgery for early gastric cancer: a non-inferiority-matched cohort study. Am J Gastroenterol. 2016;111(2):240-9.

4. Cho JH, Cha SW, Kim HG, Lee TH, Cho JY, Ko WJ, et al. Longterm outcomes of endoscopic submucosal dissection for early gastric cancer: a comparison study to surgery using propensity score-matched analysis. Surg Endosc. 2016;30(9):3762-73.

5. Ami R, Hatta W, Iijima K, Koike T, Ohkata H, Kondo Y, et al. Factors associated with metachronous gastric cancer development 
after endoscopic submucosal dissection for early gastric cancer. J Clin Gastroenterol. 2016. https://doi.org/10.1097/MCG.00000 00000000620.

6. Abe S, Oda I, Suzuki H, Nonaka S, Yoshinaga S, Nakajima $\mathrm{T}$, et al. Long-term surveillance and treatment outcomes of metachronous gastric cancer occurring after curative endoscopic submucosal dissection. Endoscopy. 2015;47(12):1113-8.

7. Kato M, Nishida T, Yamamoto K, Hayashi S, Kitamura S, Yabuta $\mathrm{T}$, et al. Scheduled endoscopic surveillance controls secondary cancer after curative endoscopic resection for early gastric cancer: a multicentre retrospective cohort study by Osaka University ESD study group. Gut. 2013;62(10):1425-32.

8. Min BH, Kim ER, Kim KM, Park CK, Lee JH, Rhee PL, et al. Surveillance strategy based on the incidence and patterns of recurrence after curative endoscopic submucosal dissection for early gastric cancer. Endoscopy. 2015;47(9):784-93.

9. Kim SB, Lee SH, Bae SI, Jeong YH, Sohn SH, Kim KO, et al. Association between Helicobacter pylori status and metachronous gastric cancer after endoscopic resection. World J Gastroenterol. 2016;22(44):9794-802.

10. Kwon YH, Heo J, Lee HS, Cho CM, Jeon SW. Failure of Helicobacter pylori eradication and age are independent risk factors for recurrent neoplasia after endoscopic resection of early gastric cancer in 283 patients. Aliment Pharmacol Ther. 2014;39(6):609-18.

11. Shin SH, da Jung H, Kim JH, Chung HS, Park JC, Shin SK, et al. Helicobacter pylori eradication prevents metachronous gastric neoplasms after endoscopic resection of gastric dysplasia. PLoS ONE. 2015;10(11):e0143257.

12. Chen HH, Lin MC, Muo CH, Yeh SY, Sung FC, Kao CH. Combination therapy of metformin and statin may decrease hepatocellular carcinoma among diabetic patients in Asia. Med (Baltim). 2015;94(24):e1013.

13. Lehman DM, Lorenzo C, Hernandez J, Wang CP. Statin use as a moderator of metformin effect on risk for prostate cancer among type 2 diabetic patients. Diabetes Care. 2012;35(5):1002-7.

14. Kautzky-Willer A, Thurner S, Klimek P. Use of statins offsets insulin-related cancer risk. J Intern Med. 2017;281(2):206-16.

15. Lee J, Lee SH, Hur KY, Woo SY, Kim SW, Kang WK. Statins and the risk of gastric cancer in diabetes patients. BMC Cancer. 2012;12:596

16. Chiu HF, Ho SC, Chang CC, Wu TN, Yang CY. Statins are associated with a reduced risk of gastric cancer: a population-based case-control study. Am J Gastroenterol. 2011;106(12):2098-103.

17. Singh PP, Singh S. Statins are associated with reduced risk of gastric cancer: a systematic review and meta-analysis. Ann Oncol. 2013;24(7):1721-30.

18. Lin CJ, Liao WC, Lin HJ, Hsu YM, Lin CL, Chen YA, et al. Statins attenuate Helicobacter pylori CagA translocation and reduce incidence of gastric cancer: in vitro and population-based case-control studies. PLoS ONE. 2016;11(1):e0146432.

19. Kim DS. Introduction: health of the health care system in Korea. Soc Work Public Health. 2010;25(2):127-41.
20. Kim DS. Special issue on the national health care system of South Korea. Soc Work Public Health. 2010;25(2):125-6.

21. National Health Insurance Service Health. Health check up. 2017. http://www.nhis.or.kr/static/html/wbd/g/a/wbdga0606.html. Accessed 8 Aug 2017

22. Jung KW, Won YJ, Oh CM, Kong HJ, Lee DH, Lee KH, et al. Cancer statistics in Korea: incidence, modality, survival, and prevalence in 2014. Cancer Res Treatment. 2017;49(2):292-305.

23. Lee SW. Cancer registration statistics, periodic statistical quality diagnosis report of 2017. Daejeon, Korea: Statistics Korea; 2017. http://kostat.go.kr/portal/eng/index.action

24. Lee YH, Han K, Ko SH, Ko KS, Lee KU. Taskforce Team of Diabetes Fact Sheet of the Korean Diabetes A. Data analytic process of a nationwide population-based study using national health information database established by national health insurance service. Diabetes Metab J. 2016;40(1):79-82.

25. Jun JK, Choi KS, Lee HY, Suh M, Park B, Song SH, et al. Effectiveness of the Korean national cancer screening program in reducing gastric cancer mortality. Gastroenterology. 2017;152(6):1319-28.e7.

26. Organization WH. International classification of diseases and related health problems: manual of the International Statistical Classification of Diseases, Injuries, and Causes of Death. Geneva: World Health Organization; 1992.

27. Organization. WH. World Health Organization. Definition and general considerations. 2017. https://www.whocc.no/ddd/definition_and_general_considera/. Accessed 2 Apr 2017.

28. Fine JP, Gray RJ. A proportional hazards model for the subdistribution of a competing risk. J Am Stat Assoc. 1999;94(446):496-509.

29. Targownik LE, Suissa S. Understanding and avoiding immortaltime bias in gastrointestinal observational research. Am J Gastroenterol. 2015;110(12):1647-50.

30. Gibot L, Follet J, Metges JP, Auvray P, Simon B, Corcos L, et al. Human caspase 7 is positively controlled by SREBP-1 and SREBP-2. Biochem J. 2009;420(3):473-83.

31. Cheng-Qian Y, Xin-Jing W, Wei X, Zhuang-Lei G, Hong-Peng Z, Songde $X$, et al. Lovastatin inhibited the growth of gastric cancer cells. Hepatogastroenterology. 2014;61(129):1-4.

32. Kim YI, Kim SY, Cho SJ, Park JH, Choi IJ, Lee YJ, et al. Longterm metformin use reduces gastric cancer risk in type 2 diabetics without insulin treatment: a nationwide cohort study. Aliment Pharmacol Ther. 2014;39(8):854-63.

Publisher's Note Springer Nature remains neutral with regard to jurisdictional claims in published maps and institutional affiliations. 